\title{
OPEN Randomised feasibility study of prehospital recognition and antibiotics for emergency patients with sepsis (PhRASe)
}

Jenna Jones ${ }^{1 凶}$, Susan Allen ${ }^{2}$, Jan Davies ${ }^{3}$, Timothy Driscoll ${ }^{1}$, Gemma Ellis², Greg Fegan ${ }^{1}$, Theresa Foster ${ }^{4}$, Nick Francis ${ }^{5}$, Saiful Islam ${ }^{1}$, Matt Morgan ${ }^{2}$, Prabath W. B. Nanayakkara ${ }^{6}$, Gavin D. Perkins ${ }^{7}$, Alison Porter ${ }^{1}$, Timothy Rainer ${ }^{2}$, Samuel Ricketts ${ }^{8}$, Bernadette Sewell ${ }^{9}$, Tracy Shanahan ${ }^{2}$, Fang Gao Smith ${ }^{10}$, Michael A. Smyth ${ }^{7}$, Helen Snooks ${ }^{1} \&$ Chris Moore $^{8}$

Severe sepsis is a time critical condition which is known to have a high mortality rate. Evidence suggests that early diagnosis and early administration of antibiotics can reduce morbidity and mortality from sepsis. The prehospital phase of emergency medical care may provide the earliest opportunity for identification of sepsis and delivery of life-saving treatment for patients. We aimed to assess the feasibility of (1) paramedics recognising and screening patients for severe sepsis, collecting blood cultures and administering intravenous antibiotics; and (2) trial methods in order to decide whether a fully-powered trial should be undertaken to determine safety and effectiveness of this intervention. Paramedics were trained in using a sepsis screening tool, aseptic blood culture collection and administration of intravenous antibiotics. If sepsis was suspected, paramedics randomly allocated patients to intervention or usual care using scratchcards. Patients were followed up at 90 days using linked anonymised data to capture length of hospital admission and mortality. We collected selfreported health-related quality of life at 90 days. We pre-specified criteria for deciding whether to progress to a fully-powered trial based on: recruitment of paramedics and patients; delivery of the intervention; retrieval of outcome data; safety; acceptability; and success of anonymised follow-up. Seventy-four of the 104 (71.2\%) eligible paramedics agreed to take part and 54 completed their training (51.9\%). Of 159 eligible patients, 146 (92\%) were recognised as eligible by study paramedics, and 118 were randomised ( $74 \%$ of eligible patients, or $81 \%$ of those recognised as eligible). Four patients subsequently dissented to be included in the trial (3\%), leaving 114 patients recruited to follow-up. All recruited patients were matched to routine data outcomes in the Secure Anonymised Information Linkage Databank. Ninety of the 114 (79\%) recruited patients had sepsis or a likely bacterial infection recorded in ED. There was no evidence of any difference between groups in patient satisfaction, and no adverse reactions reported. There were no statistically significant differences between intervention and control groups in Serious Adverse Events (ICU admissions; deaths). This feasibility study met its pre-determined progression criteria; an application will therefore be prepared and submitted for funding for a fully-powered multi-centre randomised trial.

Trial registration: ISRCTN36856873 sought 16th May 2017; https://doi.org/10.1186/ISRCTN36856873

Abbreviations

AE Adverse event

ED Emergency department

\footnotetext{
${ }^{1}$ Swansea University Medical School, ILS2, Singleton Campus, Wales SA2 8PP, UK. ${ }^{2}$ Cardiff and Vale University Health Board, Wales, UK. ${ }^{3}$ Public Contributor, Wales, UK. ${ }^{4}$ East of England Ambulance Service NHS Trust, Royston, England, UK. ${ }^{5}$ nniversity of Southampton, Southampton, UK. ${ }^{6}$ Amsterdam University Medical Centre, Amsterdam, The Netherlands. ${ }^{7}$ University of Warwick, Warwick, England, UK. ${ }^{8}$ Welsh Ambulance Services NHS Trust, Wales, UK. ${ }^{9}$ Swansea Centre for Health Economics, Swansea University, Wales, UK. ${ }^{10}$ University of Birmingham, Birmingham, England, UK. ${ }^{\bowtie}$ email: j.k.jones@swansea.ac.uk
} 


$\begin{array}{ll}\text { IV } & \text { Intravenous } \\ \text { NHS } & \text { National health service } \\ \text { PI } & \text { Principal investigator } \\ \text { PPI } & \text { Patient and public involvement } \\ \text { PRSO } & \text { Paramedic research support officer } \\ \text { RCT } & \text { Randomised controlled trial } \\ \text { SAE } & \text { SAE event } \\ \text { TMG } & \text { Trial management group } \\ \text { TSC } & \text { Trial steering committee } \\ \text { WAST } & \text { Welsh ambulance services NHS trust }\end{array}$

Background. Sepsis is a life threatening condition which requires rapid recognition and treatment in order to not only prevent death, but also to minimise the damage that is does to a person's body. Sepsis can lead to organ and limb damage, leaving patients with significant morbidity if they survive. Approximately 44,000 people die of sepsis every year in the UK ${ }^{1}$ - more than are diagnosed with bowel cancer annually ${ }^{2}$.

There is evidence that administering antibiotics early reduces complications and death from sepsis ${ }^{3-6}$. It is known that recognition of sepsis by Emergency Medical Service (EMS) personnel in the prehospital phase of care leads to faster diagnosis and treatment in the Emergency Department $(\mathrm{ED})^{7,8}$. Around half of patients with sepsis are conveyed to the ED by ambulance ${ }^{9,10}$, with an average prehospital care time of $45 \mathrm{~min}^{11}$, therefore, EMS personnel can play a significant role in the management of sepsis.

Early recognition and management of sepsis by EMS personnel offers a potentially valuable opportunity to deliver interventions early, but the evidence base to support the use of prehospital antibiotics is weak ${ }^{12,13}$. In $2016 / 17$, when this feasibility study was first conceived, there was no well-defined prehospital protocol for management of sepsis in the UK. In 2019, the Joint Royal Colleges Ambulance Liaison Committee (JRCALC), issued a prehospital clinical practice guideline for the management of sepsis, which recognises the potential value of pre-hospital sepsis screening tools, but acknowledges that, to date, none have been validated for use $\mathrm{e}^{14}$.

Aim. To assess the feasibility of paramedics screening patients for severe sepsis, collecting blood cultures and administering IV antibiotics, and to assess trial methods to inform the development of a fully-powered randomised controlled trial (RCT), to test the clinical and cost-effectiveness of prehospital IV antibiotics, if indicated.

Objectives. Intervention development. To work with clinicians, paramedics, pharmacists, and service users to develop a prehospital intervention for sepsis, comprising:

- Screening patients for sepsis

- Protocol for collection of blood cultures, administering IV antibiotics, and handover of care to ED

- Training for paramedics to deliver the agreed protocol

Intervention feasibility. To assess:

- paramedic uptake and satisfaction with the training package

- paramedic compliance with treatment protocol

- safety and acceptability of the intervention to patients and paramedics

RCT feasibility. To clarify primary and secondary outcome measures for full trial and assess:

- trial recruitment, randomisation, and data collection processes

- sample size requirements and attrition rates

- availability of outcome data

Full trial planning. To assess our findings against our progression criteria and, if met, draft a full trial protocol and application for funding.

\section{Methods}

The protocol for this feasibility study has been published ${ }^{15}$. Ethical approval was granted by Wales Research Ethics Committee 4, reference 17/WA/0186, and the trial registered on the ISRCTN database (ISRCTN36856873) on 16/05/2017. Research has been conducted in accordance with the Declaration of Helsinki. The design of the trial is a single-centre randomised parallel-group feasibility trial, with 1:1 allocation ratio. Patients were eligible if they were adult (18 years or over), with severe sepsis (see additional file for our Sepsis Screening Tool), and attended by a study paramedic within the catchment area of the participating trial hospital. Patients were excluded if they were known or thought to be pregnant, or known to be allergic to antibiotics.

The study was conducted in the Cardiff \& Vale of Glamorgan area of Wales, UK. Welsh Ambulance Service paramedics based at the five ambulance stations operating in the geographical catchment area of the University Hospital of Wales (UHW) were invited to take part. Data were collected from Patient Clinical Records (PCRs) 
completed by study paramedics, and supplemented by entries made in the PhRASe study logbook, which was secured in the resuscitation area of the ED.

Interventions. If the patient was randomly allocated to usual care, the paramedic would provide the patient with oxygen to maintain saturations over $94 \%$ (88-92\% if known to have chronic obstructive pulmonary disease) and administer $250 \mathrm{ml}$ boluses of $0.9 \%$ sodium chloride (up to $2000 \mathrm{ml}$ if the patient is hypotensive e.g. systolic blood pressure less than $100 \mathrm{mmHg}$ ). If the patient's clinical condition was judged as serious or critical, paramedics could pre-alert the receiving hospital so that the patient could be taken directly to a resuscitation bay. If the patient was randomly allocated to experimental care, usual care would be supplemented by collection of blood cultures, and IV administration of $2 \mathrm{~g}$ cefotaxime. Participating paramedics were trained in use of this new protocol in small, face to face group sessions, conducted by the Paramedic Research Support Officer (PRSO).

Patient consent. Given the potential for severe sepsis to impair mental capacity and the need to provide urgent treatment, in accordance with the Mental Capacity Act 2005, we sought approval from the Research Ethics Committee to enrol patients prior to obtaining written informed consent for the research ${ }^{16}$. At the time of paramedic attendance, therefore, patients were only asked to provide consent to treatment-to have blood cultures collected and receive antibiotics, if randomly allocated to the intervention arm.

Patients included in the feasibility trial were sent a Patient Information Leaflet, which included a 'Participant Dissent Form' at 90 days after the patient's emergency call (30 days for those recruited in the last two months of the study), offering options to either decline to receive any further correspondence from the study or to have all of their records withdrawn from the study. We included public contributors in discussions about this approach which minimises intrusion and possible distress.

Data collection. Routine ambulance service records were used for all prehospital information. Anonymised data relating to clinical outcomes and hospital diagnoses were obtained from hospital records as well as through the Secure Anonymised Information Linkage (SAIL) databank ${ }^{18}$. The SF-12 and Quality of Care Monitoring forms were sent out to participants at 90 days (30 days for participants recruited in the last two months of the study) along with the Patient Dissent Forms to be returned directly to the research team at Swansea University for data entry. Returned questionnaire responses were linked to the anonymised data for analysis within the SAIL gateway.

Adverse events. After consultation with the Trial Management Group (TMG) and Trial Steering Committee (TSC), it was decided that deaths and Intensive Care Unit (ICU) admissions were expected and would not be collected as adverse outcomes. Serious Adverse Reactions (SARs) to be monitored were agreed as:

- Anaphylaxis

- C. difficile infection

- Extravasation at the site administration of antibiotics

- Infection/cellulitis at the site of blood culture collection/administration of antibiotics

- Vascular damage at the site of blood culture collection/administration of antibiotics

Missed recruitments. As part of this feasibility study, we monitored missed recruitments in order to assess whether paramedics were able to accurately recognise patients with sepsis; whether paramedics were compliant with the protocol; and how many patients could not be randomised owing to exclusion criteria emergency.

Progression criteria. At the outset of the feasibility trial, the Trial Management Group (TMG) specified progression criteria, to be met within reasonable limits; if a progression criterion was within $5 \%$ below target we would review reasons for this and consider modifications to protocol; if within $10 \%$ we would critically review reasons for this and assess whether major changes to protocol are likely to improve the issue; if more than $10 \%$ we should consider whether progression to full trial is appropriate.

\section{Intervention feasibility.}

1. Compliance with protocol by paramedics $\rightarrow>=80 \%$ of patients recognised as eligible by study paramedics are randomly allocated to trial arm

2. Acceptability of intervention to patients - mean patient satisfaction in intervention group $>80 \%$ in the control group

3. Safety - number of patients who experience adverse events has a difference of $<10 \%$ between trial arms

4. Recognition of sepsis -

a. $>50 \%$ of patients with sepsis who are attended by study paramedics are recognised as eligible for the study

b. $>70 \%$ of randomised patients are diagnosed with sepsis in hospital 


\section{RCT Feasibility.}

5. Acceptability of RCT to paramedics $-\geq 60 \%$ of eligible paramedics agree to take part in the study.

6. Acceptability of RCT to patients - dissent to take part in the study is $\leq 30 \%$.

7. Retrieval of outcomes - follow up data for primary outcome can be collected for $\geq 70 \%$ of patients.

8. Equipoise-findings indicate that we remain in equipoise about the effectiveness of paramedic obtained blood cultures and prehospital antibiotics for sepsis.

9. Recruitment-recruitment target met to $\geq 80 \%$.

Sample size. Paramedics recruited patients over a six-month period. Based on data regarding throughput of sepsis cases in ED, we estimated recruitment of approximately 100-150 patients. In this feasibility study we did not aim to detect differences between the intervention and control groups in outcomes, but to include enough patients to assess findings against our progression criteria.

Randomisation. A randomisation schedule with a 1:1 ratio of 'intervention' or 'control', stratified by paramedic was produced by a statistician not involved in the data collection, management or analysis of the data set. A unique set of scratchcards with allocation concealed was issued to each study paramedic and kept on their person during each working shift ${ }^{19}$. When the study paramedic identified an eligible patient, they were instructed to use the next sequential scratchcard from their set out of sight of the patient. The unique number shown on the scratchcard became the patient's Study ID. The paramedic retained the scratchcard in order to store it with the randomisation log at the nurses' station in the Emergency Department, so that the Paramedic Research Support Officer (PRSO) was able to monitor randomisation. Owing to the nature of the intervention, the outcome of randomisation could not be blinded to the paramedics, patients, PRSO or data manager. The allocation was concealed from the statistician and health economist until the code for analysis was finalised.

Health economics. The health economic component of this feasibility study focused on establishing the main cost drivers by estimating the intervention implementation cost through discussions with the trial team and research paramedics, using standard unit costs for the antibiotics given. We considered a suitable framework to undertake a full cost-effectiveness analysis in a future trial.

Analysis. We analysed study data to inform progression decision and trial planning: we analysed continuous outcomes (e.g. SF-12 Score; length of stay) by t tests, or non-parametric equivalents; we report mean and standard deviation, or median and interquartile range, along with $95 \%$ confidence intervals. We analysed binary outcomes (e.g. mortality, presence of sepsis) by chi-squared tests; and report proportions and 95\% confidence intervals. We conducted exploratory analysis of potential primary outcomes to assess whether we had met progression criterion 8 (whether we remain in equipoise), and to help decide what might be the most appropriate primary outcome for a fully-powered trial. We report against each of our progression criteria to inform whether we should seek further funding for an appropriately powered trial and provide estimates for performing a power calculation for a fully-powered trial. Analysis was performed using SPSS Version 25 and reporting follows relevant CONSORT checklists ${ }^{20,21}$.

Public and patient involvement. Public and patient representatives contributed to designing, delivering, overseeing and disseminating the study. We recruited people with experience of sepsis, as either patients, carers or family members of patients, to the Trial Management Group and Trial Steering Committee. We provided briefing sessions prior to all meetings.

Changes after the study began. Owing to low response rates to study questionnaires, we submitted a substantial amendment to the Research Ethics Committee to allow us to conduct the questionnaire by telephone if the postal questionnaire had not been returned.

Ethics approval. Ethical approval was granted by Wales Research Ethics Committee 4, reference 17/ WA/0186.

\section{Results}

We report feasibility study findings in accordance with relevant CONSORT and GRIPP 2-SF checklists ${ }^{21,22}$; the CONSORT flowchart is seen in Fig. 1.

Baseline characteristics. Table 1 shows patients' baseline characteristics. Sixty-two patients (54\%) were allocated to the intervention arm. The mean age of the intervention arm was 75.6 years (range 30-99) and 38 patients (61\%) were female. The mean age of the control arm was 71.2 years (range 28-97); 33 (65\%) control participants were female. qSOFA scores (which predicts risk of in hospital mortality, with 3 being the highest risk) show that more patients had scores of 2 or 3 in the intervention group, though this wasn't statistically significant. Nine patients in the control group (18\%) and 17 in the intervention group (28\%) were already taking antibiotics at the time of their emergency call. 


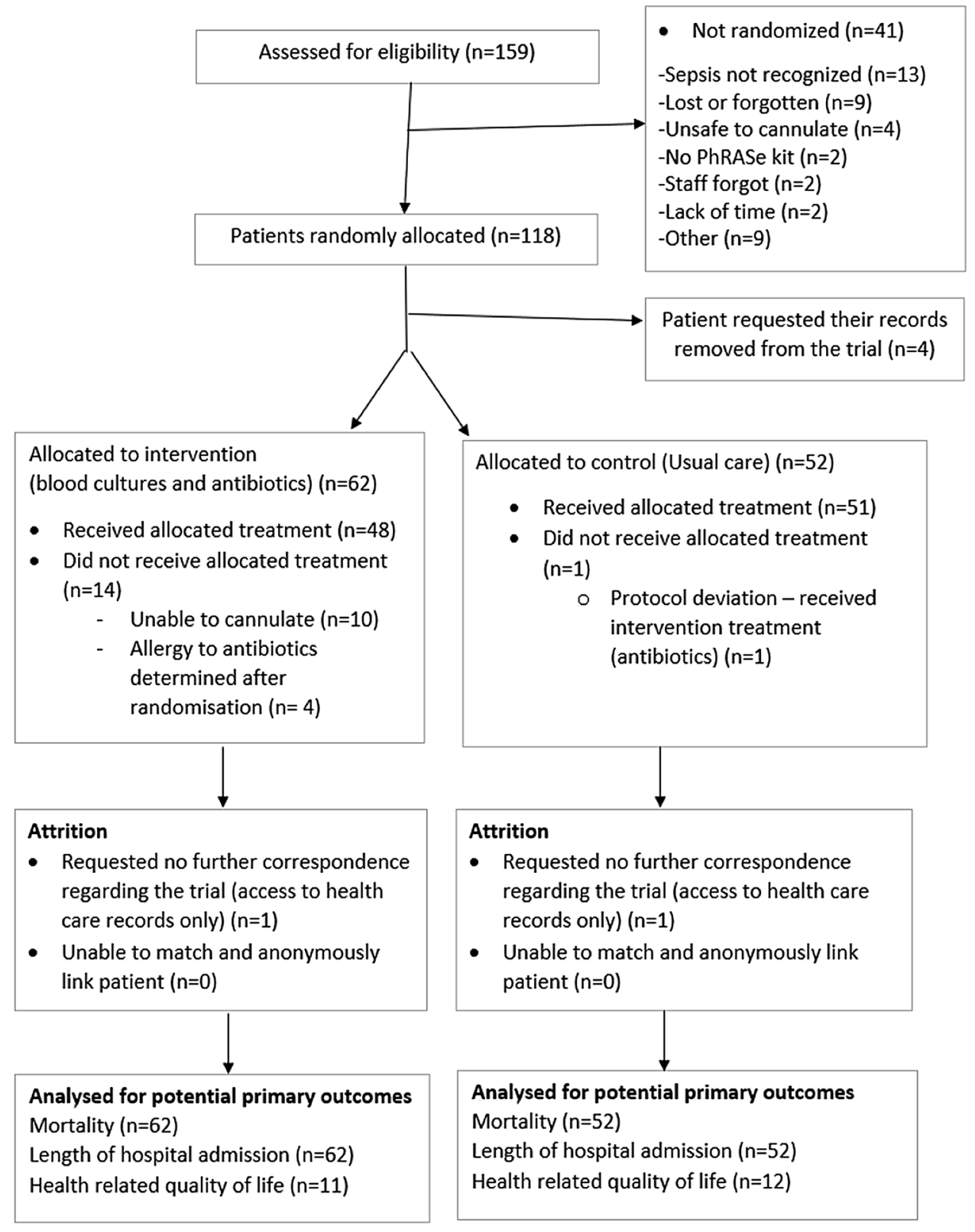

Figure 1. CONSORT flowchart.

\begin{tabular}{|l|l|l|}
\hline Characteristic & Intervention $(\mathbf{n}=\mathbf{6 2})$ & Control (n=52) \\
\hline Age in years, mean (range) & $75.6(30-99)$ & $71.2(28-97)$ \\
\hline Female, $\mathbf{n}(\%)$ & $38(61 \%)$ & $33(65 \%)$ \\
\hline qSOFA score, $\mathbf{n}(\%)$ & \multicolumn{2}{|l|}{} \\
\hline 0 & $5(8.1)$ & $2(3.8)$ \\
\hline 1 & $38(61.3)$ & $34(65.4)$ \\
\hline 2 & $15(24.2)$ & $11(21.2)$ \\
\hline 3 & $3(4.8)$ & $1(1.9)$ \\
\hline N miss (\%) & $1(1.6)$ & $4(7.7)$ \\
\hline Already taking antibiotics at emergency call, $\mathbf{n}(\%)$ & $17(28)$ & $9(18)$ \\
\hline
\end{tabular}

Table 1. Baseline characteristics. 


\begin{tabular}{|l|l|}
\hline Deviation & n \\
\hline Allergy to antibiotics identified after the patient was randomly allocated (no antibiotics given) & 4 \\
\hline Lost or damaged scratchcards & 4 \\
\hline Patient not recorded in randomisation log & 3 \\
\hline Missing kit or component & 2 \\
\hline Blood culture forms not completed & 1 \\
\hline Incomplete dose of Cefotaxime administered & 1 \\
\hline Scratchcards used out of order & 1 \\
\hline Control patient given intervention & 1 \\
\hline Patient taken to non-receiving hospital & 1 \\
\hline Total & 18 \\
\hline
\end{tabular}

Table 2. Protocol deviations.

Recruitment, consent and response rates. Seventy-four of the 104 (71.2\%) eligible paramedics who worked in the locality at the start of the study agreed to take part, and 54 (73\%) completed their training. The majority of paramedics recruited at least one patient to the trial $(n=39)$ with most of them recruiting $<=3$ patients $(n=26)$ and the maximum number of patients recruited by one paramedic being seven.

Patients were recruited from 1.12 .17 to 31.5 .18. In total, 118 patients were randomly allocated to the two trial arms; four patients subsequently dissented to be included in the trial, leaving 114 patients recruited to follow-up. Two patients (one from each arm of the trial) requested no further correspondence so we collected data from their medical records only. We matched $100 \%$ of patients to SAIL records. Twenty-three completed questionnaires were returned out of $82(28 \%)$ sent to participants who had not died before 90 days (12 of 41 (29\%) control and 11 of 41 (27\%) intervention).

The diagnoses recorded in the ED notes showed 51 of the 114 (45\%) recruited patients were diagnosed with sepsis. Thirty-nine of the $114(34 \%)$ patients were recorded as having a non-viral infection, most commonly pneumonia or urinary tract infection. Thus, 90 out of the $114(79 \%)$ recruited patients had sepsis or a likely bacterial infection recorded in ED. No serious adverse reactions were recorded.

Accuracy of recognition of sepsis by paramedics. By assessing records from the ED of patients who were treated in the resuscitation department for suspected sepsis (as documented in the ED resuscitation log book), we recorded 41 patients as 'missed recruitments' (meaning there were 159 eligible patients in total). In 13 of the missed recruitments, the patient was not recognised as having sepsis. In the other 28 missed recruitments, who were recognised as having sepsis but not randomly allocated to the trial, reasons cited included lost or forgotten scratchcards $(n=9)$; no PhRASe kit on the vehicle $(n=2)$; lack of time $(n=2)$; forgot to enter in the study $(n=2)$; unsafe/unable to cannulate $(n=4)$; and 'other' $(n=9)$. This means that 118 of 159 total eligible patients were allocated to trial arms (74\%), and 118 out of 146 patients recognised as eligible by the study paramedic were allocated to trial arms (81\%).

Protocol deviations. Eighteen protocol deviations were recorded (Table 2). All three patients who were initially not recorded in the randomisation log were identified from WAST clinical records and included in follow up. One patient was taken to a non-study hospital as a result of a hospital divert protocol being implemented during a period of high demand at the ED.

Health economics. Intervention implementation costs included paramedic training costs and total callout costs (including antibiotics and blood cultures in the intervention group). Fifty-four paramedics were trained in two 1.5-h sessions (online and face-to-face training) at a total cost of $£ 8,128.50$. This amounts to $£ 68.89$ per trial participant $(\mathrm{n}=118)$. Per-patient training costs are subject to economies of scale and maydecrease if the intervention is on a larger scale. Additionally, total callout costs (comprising staff cost based on time from callout to arrival at hospital, cost of cefotaxime $2 \mathrm{~g}$ for intravenous administration ${ }^{16}$ and laboratory cost of blood culture ${ }^{17}$ were $£ 23.33$ ( $95 \% \mathrm{CI}:-2.71$ to 49.36 ; $\mathrm{p}=0.09$ ) higher in the intervention group, based on the additional drug and culture costs and an on average $10.2 \mathrm{~min}(95 \% \mathrm{CI}-9.0$ to $29.4 ; \mathrm{p}=0.11)$ longer job cycle time.

Healthcare resource use data linked through the SAIL databank were available for all participants and showed that the main cost drivers can be expected to be inpatient admissions and ICU stays (Table 1). There was evidence of significant missing SF-12 data with only 23 patients (20.18\%) returning completed questionnaires at 90 days.

Outcome analyses. Unadjusted comparisons (seen in Table 3) between deaths; ICU admissions; ED attendances in the three months following the initial emergency call; length of hospital admission; job cycle and on-scene times satisfaction with care; and health-related quality of life showed no differences at the $\mathrm{P}=0.05$ level of significance. The only statistically significant difference between trial arms was the number of hospital admissions in the three months following the initial emergency call (87 in the experimental arm compared with 56 in the usual care arm, $\mathrm{p}=0.0002$ ). 


\begin{tabular}{|c|c|c|c|c|}
\hline Outcome & Intervention $(n=62)$ & Control $(n=52)$ & Total $(\mathrm{N}=114)$ & Difference (95\% CI, Significance level) \\
\hline \multicolumn{5}{|l|}{ Routine data (SAIL) } \\
\hline 90 day Mortality n(\%) & $21(33.9)$ & $11(21.2)$ & $32(28.1)$ & $\begin{array}{l}\text { Odds ratio } \\
1.9(0.82,4.5) \\
p=0.13\end{array}$ \\
\hline ICU admissions (yes) (for primary emergency call), n (\%) & $5(8.1)$ & $1(1.9)$ & $6(5.3)$ & $\begin{array}{l}\text { Odds ratio: } \\
4.5(0.51,39.6) \\
p=0.14\end{array}$ \\
\hline $\begin{array}{l}\text { Number of ED attendances up to } 90 \text { days from emergency call (mean, SD } \\
\text { of ED attendance per patient) }\end{array}$ & $79(1.3,0.5)$ & $76(1.5,0.7)$ & $155(1.4,0.6)$ & $\begin{array}{l}\text { Mean Diff } \\
-0.2(-0.41,0.03), p=0.1\end{array}$ \\
\hline $\begin{array}{l}\text { Number of hospital admissions up to } 90 \text { days from emergency call } \\
\text { (mean, SD admission per patient) }\end{array}$ & $87(3.5,3.3)$ & $56(1.8,1.0)$ & $143(2.83,2.7)$ & $\begin{array}{l}\text { Mean Diff } \\
1.8(1.0,2.5) \cdot p<0.05\end{array}$ \\
\hline $\begin{array}{l}\text { Bed days used up to } 90 \text { days from emergency call (mean }(95 \% \mathrm{CI}) \text {, } \\
\text { median, sd) }\end{array}$ & $14.2(8.8,19.5) ; 5,25.2$ & $\begin{array}{l}14.4(7.3,21.5) \\
4,26.5\end{array}$ & $\begin{array}{l}14.3(10.0,18.5) \\
4,25.6\end{array}$ & $-0.3(-8.9,8.5), p=0.9$ \\
\hline \multicolumn{5}{|l|}{ Data collected by PRSO } \\
\hline $\begin{array}{l}\text { Time interval (minutes) from emergency call to administration of antibi- } \\
\text { otic m, sd, nmiss }(\%)\end{array}$ & $131,147,15(24.2)$ & NA & NA & NA \\
\hline $\begin{array}{l}\text { Job cycle time (minutes) from time of call to arrival at hospital } \mathrm{m}, \mathrm{sd} \text {, } \\
\text { nmiss }(\%)\end{array}$ & $155,132,0$ & $136,87.5,0$ & $146,114,0$ & $\begin{array}{l}18.7(-23,9,61.3) \\
p=0.2\end{array}$ \\
\hline On scene time (minutes) m, sd & $77.2,131$ & $65.5,86.9$ & $71.9,113$ & $\begin{array}{l}11.7(-30.5,53.9) \\
p=0.3\end{array}$ \\
\hline Blood Culture received & 48 & NA & NA & NA \\
\hline Contamination of blood cultures & & NA & NA & NA \\
\hline Yes & $1(2.1)$ & & & \\
\hline No & $42(87.5)$ & & & \\
\hline Missing & 0 & & & \\
\hline Not possible to identify & $5(10.4)$ & & & \\
\hline \multicolumn{5}{|l|}{ Patient reported outcome measures } \\
\hline Quality of care monitor score & $\begin{array}{l}\mathrm{n}=6 \\
39.7,5.6\end{array}$ & $\begin{array}{l}\mathrm{n}=9 \\
39.7,8.3\end{array}$ & $\begin{array}{l}\mathrm{n}=15 \\
39.7,6.6\end{array}$ & $\begin{array}{l}\text { Mean diff: } \\
0,(-7.8,7.8), p=1.0\end{array}$ \\
\hline SF-12 score (at 90 days) & $\mathrm{n}=11$ & $\mathrm{n}=12$ & $\mathrm{n}=23$ & \\
\hline Physical component score & $31.6(11.2)$ & $35.3(12.6)$ & $33.5(11.8)$ & $3.6,(-6.7,13.9), p=0.5$ \\
\hline $\begin{array}{l}\text { Mental component score } \\
\text { Mean (SD) }\end{array}$ & $41.8(14.5)$ & $46.5(15.7)$ & $44.3(14.9)$ & $\begin{array}{l}4.7,(-8.5,17.8) \\
p=0.5\end{array}$ \\
\hline
\end{tabular}

Table 3. Unadjusted analysis of outcomes between groups. $M$ mean, med median, min minimum, $\max$ maximum, $s d$ standard deviation, $n$ miss number of missing.

Sample size and recruitment required for a full trial. We have calculated the sample size required to find a $5 \%$ difference in mortality between groups, using $90 \%$ power and $5 \%$ significance, a difference agreed by the research team including PPI representatives and clinicians as important. Based on a 90 day mortality rate of approximately $28 \%$ in this feasibility trial, 3200 patients are needed in analysis. If approximately $10 \%$ of participants dissent from anonymised follow up of routine records, and we are unable to match $1 \%$ of cases in routine health data sources, we will need to randomly allocate 3591 patients. Using the PhRASe rate of recruitment and data on sepsis related calls from potential collaborating ambulance services, we will need five sites to each recruit approximately 718 patients during a 24 month recruitment period.

Results summary-Fulfilment of Progression Criteria. This feasibility study met all of its pre-determined progression criteria, as summarised in Table 4.

\section{Discussion}

The PhRASe feasibility study achieved all of its progression criteria within reasonable limits (Table 4).

With regard to acceptability of the RCT to paramedics, more paramedics initially expressed an interest in taking part than completed training. It is not known why this was; we would need to consider what the barriers to participation were in order to assess whether this could be improved upon in a definitive trial. Though this feasibility trial invited paramedics to volunteer, we would in future try to recruit all paramedics in order to meet the required sample size.

As there was a $13 \%$ absolute difference in 90 -day mortality between trial arms, we recommend that in a definitive trial mortality is monitored continuously, and reported on a monthly basis, to the independent Data Monitoring Committee.

On four occasions, patients were entered into the trial and subsequently found to have an allergy to antibiotics. We would have to further emphasise in training for a definitive trial that the patient's drug allergies should be checked prior to patient recruitment.

Although the recently published PHANTASi trial ${ }^{23}$ of prehospital antibiotics in the Netherlands found that nurses giving antibiotics in the ambulance did not lead to improved survival, there are factors which make it 


\begin{tabular}{|c|c|c|}
\hline Progression criterion & Relevant result & Interpretation \\
\hline $\begin{array}{l}\text { 1: Compliance with protocol by paramedics-no less than } 80 \% \text { of } \\
\text { patients recognised as eligible patients by study paramedics are } \\
\text { randomised }\end{array}$ & $\begin{array}{l}118 \text { of } 146(81 \%) \text { eligible patients were recruited and randomly } \\
\text { allocated to trial arm }\end{array}$ & Criterion met \\
\hline $\begin{array}{l}\text { 2: Acceptability of intervention to patients-mean patient satisfac- } \\
\text { tion in intervention group is not less than } 80 \% \text { of patient satisfaction } \\
\text { in the control group }\end{array}$ & $\begin{array}{l}\text { There was no evidence of any difference between groups in patient } \\
\text { satisfaction }\end{array}$ & Criterion met \\
\hline $\begin{array}{l}\text { 3: Safety-number of patients who experience adverse events has a } \\
\text { difference of less than } 10 \% \text { between trial arms }\end{array}$ & $\begin{array}{l}\text { No adverse reactions were reported. There were no statistically } \\
\text { significant differences between intervention and control groups in } \\
\text { Serious Adverse Events (ICU admissions; deaths) }\end{array}$ & Criterion met \\
\hline $\begin{array}{l}\text { 4: Recognition of sepsis-a. at least } 50 \% \text { of patients with suspected } \\
\text { sepsis who are attended by study paramedics are recognised as } \\
\text { eligible for the study }\end{array}$ & $\begin{array}{l}118 \text { of } 159(74 \%) \text { patients who were recorded as having sepsis in ED } \\
\text { were allocated to trial arms }\end{array}$ & Criterion met \\
\hline $\begin{array}{l}\text { 4: Recognition of sepsis-b. at least } 70 \% \text { of randomised patients are } \\
\text { diagnosed with sepsis in hospital }\end{array}$ & $\begin{array}{l}\text { Ninety of } 114(79 \%) \text { recruited patients were recorded as having } \\
\text { sepsis or a likely bacterial infection }\end{array}$ & Criterion met \\
\hline $\begin{array}{l}\text { 5: Acceptability of RCT to paramedics-at least } 60 \% \text { of eligible } \\
\text { paramedics agree to take part in the study }\end{array}$ & $\begin{array}{l}\text { Seventy-four of } 104 \text { (71\%) eligible paramedics who work in the Car- } \\
\text { diff and Vale locality expressed an interest to take part in PhRASe. } \\
\text { Fifty-four paramedics completed their training }(51.9 \%)\end{array}$ & Criterion met within reasonable limits \\
\hline $\begin{array}{l}\text { 6: Acceptability of RCT to patients-dissent from taking part in the } \\
\text { study is } 30 \% \text { or less }\end{array}$ & Dissent to take part in the study was $3 \%(4 / 118)$ & Criterion met \\
\hline $\begin{array}{l}\text { 7: Retrieval of outcomes-follow up data for primary outcome } \\
\text { suitable for fully-powered trial can be collected for } 70 \% \text { or more of } \\
\text { patients }\end{array}$ & $\begin{array}{l}\text { All recruited patients were matched to routine data outcomes in } \\
\text { SAIL }\end{array}$ & Criterion met \\
\hline $\begin{array}{l}\text { 8: Equipoise-findings indicate that we remain in equipoise about } \\
\text { the effectiveness of paramedic obtained blood cultures and prehospi- } \\
\text { tal antibiotics for sepsis }\end{array}$ & $\begin{array}{l}\text { Only one outcome (number of hospital admissions in three months } \\
\text { following emergency call) was statistically significantly different } \\
\text { between trial arms }\end{array}$ & Criterion met within reasonable limits \\
\hline 10. Recruitment-recruitment target met to at least $80 \%$ & $\begin{array}{l}\text { We allocated } 118 \text { patients to trial arms, with } 114 \text { ultimately recruited. } \\
\text { (Our aim was to recruit } 100-150 \text { patients) }\end{array}$ & Criterion met \\
\hline
\end{tabular}

Table 4. Summary of results against progression criteria.

different to our proposed study. The PHANTASi trial included patients with all severities of sepsis; the overall mortality rate was just $8 \%$ at 28 days (compared to $28 \%$ at 90 days in this feasibility study). The study was therefore underpowered to detect a difference in mortality in only those patients with severe sepsis. Ambulances in the Netherlands are staffed by nurses with years of experience in treating critically ill patients and who have followed additional specialised training before applying to qualify as a registered ambulance nurse. This research team proposes to conduct a randomised trial for patients with more severe illness, in an EMS system staffed by paramedics rather than nurses.

Weinberger et al. ${ }^{24}$ discuss the difficulties in assessing time to first antibiotics in patients with sepsis, as patients contact emergency services (or their family doctor or out of hours services) at different time points in their illness. Though we accept this is true, as we plan to conduct an RCT, the range of time patients' have waited before calling emergency services should not systematically vary between groups. Weinberger et al. also argue that patients with multiple comorbidities may not present with typical symptoms of sepsis, as would an otherwise healthy person, which could prolong their wait for antibiotics. Again, there is no reason to believe one randomised group would have significantly more unwell patients in it, but, for this reason, we would ensure comorbidities are included as covariates in the multivariate analysis of outcomes in the full RCT.

Limitations. Patient quality of life data using the SF-12 questionnaire were only obtained from 23 of 82 eligible participants (28\%). While this had to be expected considering the health status of the population in question, the lack of response to the SF-12 quality of life questionnaire would need to be addressed in order to undertake a cost-utility analysis. This may include investigating barriers for questionnaire completion and putting into place measure to improve return rate of outcome measures.

Interpretation and conclusion. We compared trial arms only to confirm that we remain in equipoise about the clinical effectiveness of pre-hospital antibiotics for sepsis administered by paramedics. Hence, we treat observed differences in outcomes with caution in this feasibility trial.

This feasibility study met its pre-determined progression criteria; an application will therefore be prepared and submitted for funding for a fully-powered multi-centre randomised trial.

Trial status. Complete and reported to the funder in June 2019.

\section{Data availability}

Study data is available upon request.

Received: 28 April 2021; Accepted: 17 August 2021

Published online: 20 September 2021 


\section{References}

1. Sepsis Trust UK Website https://sepsistrust.org/about/about-sepsis/ Accessed 01/04/21

2. Cancer Research UK Website https://www.cancerresearchuk.org/health-professional/cancer-statistics/incidence/common-cance rs-compared\#heading-Zero Accessed 01/04/21

3. Daniels, R. Surviving the first hours in sepsis: Getting the basics right (an intensivist's perspective). J. Antimicrob. Chemoth. 66(2), 11-23 (2011).

4. Daniels, R., Nutbeam, T., McNamara, G. \& Galvin, C. The sepsis six and the severe sepsis resuscitation bundle: A prospective observational cohort study. Emerg. Med. J. 28(6), 507-512 (2011).

5. Kumar, A. et al. Duration of hypotension before initiation of effective antimicrobial therapy is the critical determinant of survival in human septic shock. Crit. Care Med. 34(6), 1589-1596 (2006).

6. Ferrer, R. et al. Empiric antibiotic treatment reduces mortality in severe sepsis and septic shock from the first hour: Results from a guideline-based performance improvement program. Crit. Care Med. 42(8), 1749-1755 (2014).

7. Studnek, J. R., Artho, M. R., Garner, C. L. \& Jones, A. E. The impact of emergency medical services on the ED care of severe sepsis. Am. J. Emerg. Med. 30(1), 51-56 (2012).

8. Seymour, C. W. et al. Out-ofhospital characteristics and care of patients with severe sepsis: A cohort study. J. Crit. Care. 25(4), 553-562 (2010).

9. Gray, A. et al. The epidemiology of adults with severe sepsis and septic shock in Scottish emergency departments. Emerg. Med. J. 30(5), 397-401 (2013).

10. Herlitz, J. et al. Suspicion and treatment of severe sepsis. An overview of the prehospital chain of care. Scand. J. Trauma Resusc. Emerg. Med. 27(20), 42 (2012)

11. Seymour, C. W. et al. Severe sepsis in pre-hospital emergency care: Analysis of incidence, care, and outcome. Am. J. Respir. Crit. Care Med. 186(12), 1264-1271 (2012).

12. Travers, A., Green, R., Cain, E., Campbell, S.G., Jensen, J.L., Petrie, D. Can paramedics diagnose sepsis in the prehospital setting? A feasibility study. Poster at Canadian Association of Emergency Physicians Annual Conference; 2013 June 1-5; Vancouver, Canada

13. Chamberlain D. Prehospital administered intravenous antimicrobial protocol for septic shock: a prospective randomized clinical trial. Poster session presented at: Intensive Care and Emergency Medicine 29th International Symposium; 2009 March 24-27; Brussels, Belgium

14. Brown, S. N., Kumar, D. S., James, C., \& Mark, J. (Eds.). (2019). JRCALC clinical guidelines 2019. Bridgwater: Class Professional.

15. Moore, C. et al. Prehospital recognition and antibiotics for emergency patients with sepsis: Protocol for a feasibility study. Pilot Feasib. Stud. 4, 64. https://doi.org/10.1186/s40814-018-0258-8 (2018).

16. General Medical Council. Good Practice in Research and Consent to Research. London: GMC; 2010. http://www.gmc-uk.org/ Good_practice_in_research_and_consent_to_research.pdf_58834843.pdf Accessed 21/02/18.

17. SAIL databank https://saildatabank.com/ Accessed 12/8/21

18. Snooks, H. A. et al. Support and Assessment for Fall Emergency Referrals (SAFER) 2: A cluster randomised trial and systematic review of clinical effectiveness and cost-effectiveness of new protocols for emergency ambulance paramedics to assess older people following a fall with referral to community-based care when appropriate. Health Technol. Assess. 21(13), 1-218 (2017).

19. Keen, L. et al. Use of scratchcards for allocation concealment in a prehospital randomised controlled trial. Emerg. Med. J. 35(11), 708-710. https://doi.org/10.1136/emermed-2018-207881 (2018).

20. Chan, A.-W., Tetzlaff, J., Altman, D., Dickersin, K. \& Moher, D. SPIRIT: New guidance for content of clinical trial protocols. Lancet 381, 91-92 (2013)

21. Schulz, K.F., Altman, D.G., Moher, D., for the CONSORT Group. CONSORT. Statement: updated guidelines for reporting parallel group randomised trials. BMJ 2010(340), 332 (2010).

22. Staniszewska, S. et al. GRIPP2 reporting checklists: Tools to improve reporting of patient and public involvement in research. $B M J$ 358, j3453 (2017).

23. Alam, N. et al. Prehospital antibiotics in the ambulance for sepsis: A multicentre, open label, randomised trial. Lancet Respir. Med. 6(1), 40-50 (2018)

24. Weinberger, J., Rhee, C. \& Klompas, M. A critical analysis of the literature on time-to-antibiotics in suspected sepsis. J. Infect. Dis. 222, S110-S118 (2020).

\section{Acknowledgements}

We extend our sincere gratitude to all the paramedics who volunteered to take part in PhRASe, and gave their own time to undertake training. We thank the members of the Trial Steering Committee. We also wish to thank Emma Baker and Angharad Claydon, Project Administrators, for their hard work and valuable contribution to this feasibility study.

\section{Author contributions}

J.J. prepared the manuscript. Subsequent drafts received editorial input from all the authors. C.M. conceived and developed the research with methodological advice from H.A.S. B.S. designed and led the health economic component of the study. S.I. designed and led the statistical analysis of the study. A.P. designed and led the qualitative component of the study All authors read and approved the final manuscript.

\section{Funding}

PhRASe was funded by Health and Care Research Wales through their Research for Patient and Public Benefit (RfPPB) stream. Reference number 1191. PhRASe was sponsored by Dr Brendan Lloyd, Medical Director of the Welsh Ambulance Services NHS Trust.

\section{Competing interests}

The authors declare no competing interests.

\section{Additional information}

Supplementary Information The online version contains supplementary material available at https://doi.org/ 10.1038/s41598-021-97979-w.

Correspondence and requests for materials should be addressed to J.J.

Reprints and permissions information is available at www.nature.com/reprints. 
Publisher's note Springer Nature remains neutral with regard to jurisdictional claims in published maps and institutional affiliations.

(c) (i) Open Access This article is licensed under a Creative Commons Attribution 4.0 International License, which permits use, sharing, adaptation, distribution and reproduction in any medium or format, as long as you give appropriate credit to the original author(s) and the source, provide a link to the Creative Commons licence, and indicate if changes were made. The images or other third party material in this article are included in the article's Creative Commons licence, unless indicated otherwise in a credit line to the material. If material is not included in the article's Creative Commons licence and your intended use is not permitted by statutory regulation or exceeds the permitted use, you will need to obtain permission directly from the copyright holder. To view a copy of this licence, visit http://creativecommons.org/licenses/by/4.0/.

(C) The Author(s) 2021, corrected publication 2022 